PART 3

REPORTS OF MEETINGS OF COMMISSIONS

COMPTES RENDUS DES SÉANCES DES COMMISSIONS 


\title{
COMMISSION 4: EPHEMERIDES (EPHÉMÉRIDES)
}

\author{
Report of Meetings, 21 and 29 August 1973
}

President: J. Kovalevsky.

SECRETARY: B. Morando.

At the opening of the first session the chairman regretted that two prominent members of the Commission, Mr Sadler and Dr Duncombe were unable to attend. Greetings from the members of the Commission were conveyed to both by the Chairman.

Dr Morando was appointed as Secretary for both sessions of Commission 4.

\section{Membership}

Next president will be the past vice-president Dr Duncombe, the new vice-president Dr V K. Abalakin. The Organizing Committee will include: J. Kovalevsky, T. Lederle, B. Morando, D. Mulholland, A. M. Sinzi, G. Wilkins.

New members: There is one retiring member Dr Sato. He will be replaced by Dr S. Aoki. Dr F. Gondolatsch has retired from the Astronomisches Rechen-Institut but, as he is still active, he was asked not to retire from the Commission. Dr Williams from JPL, proposed as member of the IAU by the U.S. National Committee, has been made a member of the Commission.

J. H. Lieske, C. Osterwinter and H. G. Walter who were already consulting members and were proposed members of the IAU, were made members of the Commission.

It was decided that there will be no consulting member of the Commission for the next three years.

\section{Report of the Commission}

In order to comply with the wish of the General Secretary of the Union, the Chairman of Commission 4 was not allowed to increase the length of the report. So it was that the reports of the directors of the almanac offices could not be included in full. Dr Wilkins remarked that the directors should have been asked to write shorter reports that could have been included in the final report.

The report of the commission as published in Volume 15A was formally adopted. The Commission expressed the wish that the publication of the commission reports be continued, and it is wished that the length of the report of Commission 4 should not be further reduced.

\section{Report of the Working Group on Numerical Data}

This group was set up at the last General Assembly in Brighton. The field of ephemerides is covered by the International Information Bureau on Astronomical Ephemerides. The group has not yet met as a group but will do so at the time of the present $\mathrm{G}$. A.

\section{Report of the BIIEA}

Dr Morando, director of the International Information Bureau on Astronomical Ephemerides, (BIIEA) gave a very short report on the activity of the Bureau since the last General Assenbly of the IAU when it was created. A more complete report was given at a public meeting of the Scientific Advisory Council of BIIEA on August 22nd.

A resolution was adopted asking an increased financial support of the BIIEA by the IAU. It was later decided by the general assembly that this support will be 880 U.S. dollars for the three years to come. 


\section{Reports and Future of Working Groups}

The reports on the work of these working groups were the main topic of the IAU Joint Discussion $\mathrm{N}^{\circ} 1$ and will be published in the Proceedings of this discussion. All three working groups considered that they have not reached final decisions. The commission decided that the three working groups should continue their work for the next three years. 1976 is a deadline for decisions to be taken if it is wished that they come into effect not later than 1980.

J. Kovalevsky conveyed R. L. Duncombe's invitation, supported by Dr Strand, to hold a symposium on astronomical constants in Washington in 1974. This invitation was gratefully accepted, but it was suggested that the attendance be restricted to very few active members of the working groups and the heads of the almanac offices who will have to apply the decisions. It was also agreed that the chairman of a WG may invite new people to the group if it is agreeable to the presidents of the commissions involved.

\section{Priorities for Future Research}

A long general discussion was held on this problem and the following summary, drafted for the second session was formally adopted.

'The goal of Commission 4 is to provide ephemerides to the users, with the required precision. This is a rather technical matter and involves essentially research in methods, generally related to Celestial Mechanics.

However, Commission 4 has a good knowledge of the needs expressed by the users and can therefore make some recommendation on the most urgent research.

These are the following. (The order is not indicative of any relative priority.)

- To obtain and adopt the best possible self consistent reference set of astronomical constants (angular constants, masses, time reference).

- To obtain much better ephemerides of natural satellites, in the first place, the Galilean satellites of Jupiter and the 7 first satellites of Saturn, for some will soon be visited by space probes.

- To improve in a general way the celestial system of reference and to bind it as well as possible to the terrestrial system.

- To obtain new planetary theories with the new system of masses.

The researches implied to meet these objectives are self-evident and the need of subsequent observations is to be stressed'

Dr Morando suggested that a resolution be passed on the desirability of having good observations of the natural satellites of the planets. At the second session, the resolution prepared jointly by a small committee of Commissions 20 and 4 and already adopted by Commission 20 was unanimously adopted.

It reads as follows:

'Noting that the ephemerides of most of the natural satellites are based on observations made long ago, and that recent efforts to improve these ephemerides have been greatly hampered by the extreme paucity of recent observations;

Acknowledging that much higher accuracy in the ephemerides of these objects will be required in the near future, not only for earth-based studies, but also for the future exploration of the outer solar system by spacecrafts.

Commissions 4 and 20 urge that a reasonable amount of telescope time be made available for observations useful for the improvement of such ephemerides'

Commission 4 also unanimously supported Commission's 8 resolution on the improvement of FK4, the addition of faint stars to the catalogue towards the building of FK5. It reads as follows:

'It is recommended that an improvement of FK4 and its extension to a fainter magnitude limit, resulting in a new fundamental catalogue, the FK5, be carried out at the Astronomisches RechenInstitut, Heidelberg; that observatories throughout the world contribute to this project by providing basic observations, on punched cards if possible; and that all information pertinent to the formation of the FK 5 be transmitted to the Astronomisches Rechen-Institut with the observations.' 


\section{Physical Ephemeris of Mars}

A proposed resolution on new parameters for the physical ephemeris of Mars has been set up. It was explained by Dr de Vaucouleurs. The period of rotation has been improved by Ashbrook in 1953, and Mariner 9 has given better knowledge of the planet. Definitive landmarks being available on the surface of the planet, time has come for a new set of parameters to be used after 1979. (Ref. G. de Vaucouleurs et al., J. Geophys. Res. 78, No. 20, 4395, July 1973.)

This resolution reads as follows:

'Considering that during the past three years new determinations of the elements of Mars have indicated the need for a revision of the elements currently adopted in the physical ephemeris of Mars and that a new definition of the origin of the areographic longitudes has been proposed for the Mariner 9 cartographic products (G. de Vaucouleurs, M. E. Davies and F. M. Sturms, Jr., J. Geophys. Res., in press),

\section{Commission 4 recommends:}

(1) that the new elements and new definition of the origin of the areographic longitudes given below be incorporated in the physical ephemeris of Mars after 1979 or as soon as deemed practicable in the judgement of the cognizant Directors of the National Ephemeris Offices, but not before the tie between the new and current systems has been firmly established by appropriate comparisons between the ground-based and Mariner 9 coordinate systems.

The new elernents are as follows:

1. Celestial coordinates of North Pole of Mars (referred to the 1950.0 equinox).

$$
\begin{aligned}
& \alpha_{0}=317.32-0.1011 T \\
& \delta_{0}=+52.68-0.0570 T,
\end{aligned}
$$

where the second terms are the Mars precession rates and $T$ is measured in Julian centuries from JD 2433282.5 (Jan 0 1950.0) The corresponding inclination of the Mars equator to the Mars orbit is

$$
I=25: 19969+0.01219 T+0.00006 T^{2} .
$$

2. Angle measured along the Mars equator from the ascending node on the mean 1950.0 Earth equator to the Mars autumnal equinox

$$
\Delta_{50}=42: 93538-0.09040 T-0.00010 T^{2} .
$$

3. Angle from the Mars vernal equinox to the Mars prime meridian.

$$
V=148: 24+350: 892017 \text { (JD }-2433282.5) \text {, }
$$

where the constant term is consistent with the new definition of the origin of areographic longitudes by the center of a small crater (Airy-0) and the rotation rate with a new determination of the rotation period (de Vaucouleurs et al., loc. cit.). $V_{0}$ may require a small zero point correction when the relation between coordınates in the current ephemeris and in the Mariner 9 system has been finally established (Davies and de Vaucouleurs, work in progress).

(2) that the present investigations be continued in order to establish precisely the tie between the new and the current systems, and

(3) that a recomputation of the physical ephemeris based on these new elements be prepared for the years 1800 to $1979 . '$

It was remarked that $V_{0}=148^{\circ} 24$ might be a provisional value and that the usual quantity $\varphi_{0}$ is 
equal to $\Delta_{50}+180^{\circ}$. It would be useful to compute the ephemeris back to the first observation of landmarks by Cassini in 1672 .

The resolution was unanimously accepted after some remarks from Dr O'Handley, Dr Wilkins and Dr Mulholland from which it appeared that the change to be introduced will be less than $1 / 10$ th of a degree in longitude $(5 \mathrm{~km})$. It appeared also that the radar resolution is at most $10 \mathrm{~km}$.

\section{Future Changes in the National Ephemerides}

Dr Kovalevsky stated how things stand. There are three users:

(1) Connaissance des Temps, American Ephemeris etc...users. They seem to be satisfied with what is done for them now.

(2) People who want to know roughly where the bodies are (amateurs, observers that wish to choose among predictable events etc...).

(3) People that need ephemerides very much in advance. For these people data like the Advanced proofs of the AE, or special ephemerides for a given body could be useful.

In a long letter, Dr. Everhart, explained that he would prefer that no precise values of $\alpha, \delta$ be published, they should be good enough to settle the telescope. Dr Morando asked whether 1' would be agreeable. Dr de Vaucouleurs replied that, as night assistants in observatories have generally a primary education, they should not have to do any calculations. Dr de Vaucouleurs thinks that a precision of 1 " would be desirable, which means that the amount of data to be published by the almanac offices would still be very bulky.

On the suggestion that computer printout sent to users would be good enough and would save printing, it was remarked that it would be just as expensive if not more. Dr Kovalevsky, in his conclusion of the discussion feels that more efforts in consulting users should be deployed.

\section{New Definition of TAI}

In view of the conclusions reached at the joint meetings of Commission 4 and 31 , it was suggested that the new President should collect the feelings of the members of the commission on the desirability of changing the definition of TAI (or stepping it in the future) by an amount of exactly 32 seconds. 